\title{
Toward rapamycin analog (rapalog)-based precision cancer therapy
}

\author{
Ling-hua MENG ${ }^{1,2}$, XF Steven ZHENG ${ }^{1, *}$
}

\begin{abstract}
${ }^{1}$ Rutgers Cancer Institute of New Jersey and Division of Cancer Pharmacology, Robert Wood Johnson Medical School Rutgers, The State University of New Jersey, New Brunswick, NJ 08903, USA; ${ }^{2}$ Division of Antitumor Pharmacology, State Key Laboratory of Drug Research, Shanghai Institute of Materia Medica, Chinese Academy of Sciences, Shanghai 201203, China
\end{abstract}

\begin{abstract}
Rapamycin and its analogs (rapalogs) are the first generation of mTOR inhibitors, which have the same molecular scaffold, but different physiochemical properties. Rapalogs are being tested in a wide spectrum of human tumors as both monotherapy and a component of combination therapy. Among them, temsirolimus and everolimus have been approved for the treatment of breast and renal cancer. However, objective response rates with rapalogs in clinical trials are modest and variable. Identification of biomarkers predicting response to rapalogs, and discovery of drug combinations with improved efficacy and tolerated toxicity are critical to moving this class of targeted therapeutics forward. This review focuses on the aberrations in the PI3K/mTOR pathway in human tumor cells or tissues as predictive biomarkers for rapalog efficacy. Recent results of combinational therapy using rapalogs and other anticancer drugs are documented. With the rapid development of next-generation genomic sequencing and precision medicine, rapalogs will provide greater benefits to cancer patients.
\end{abstract}

Keywords: rapalog; rapamycin; mTOR; precision cancer therapy

Acta Pharmacologica Sinica (2015) 36: 1163-1169; doi: 10.1038/aps.2015.68; published online 24 Aug 2015

\section{Introduction}

The serine/threonine kinase mTOR (mechanistic target of rapamycin) is at the center of signaling pathways that are critical for the regulation of cellular metabolism, growth, and proliferation $^{[1]}$. mTOR forms at least two separate protein complexes: mTORC1 and mTORC2. mTORC1 is composed of mTOR, raptor, mLST8 and deptor ${ }^{[2,3]}$. Some of the mTORC1 components (mTOR, mLST8 and deptor) are also present in mTORC2. mTORC2 contains several unique subunits, including rictor, $\mathrm{mSIN} 1$ and PRR $5^{[4]}$. The best-known substrates of mTORC1 are S6 kinases (S6K1 and S6K2) and 4E-BP1 (eukaryotic initiation factor 4E-binding protein-1), which are involved in protein translation ${ }^{[1]}$. To coordinate cell growth and proliferation, mTORC1 responds not only to growth factors but also to energy, amino acids and oxygen levels. mTOR signaling is aberrantly hyper-activated with high frequency in various types of cancers and has been shown to be an important target for cancer therapy.

Rapamycin and its analogs are recognized as the first generation of mTOR inhibitors, which inhibit the activity of

\footnotetext{
* To whom correspondence should be addressed. E-mail zhengst@cinj.rutgers.edu (X. F. Steven ZHENG)

Received 2015-04-14 Accepted 2015-06-12
}

mTORC1 via binding to FKBP-12 and forming a ternary complex with mTOR. Derivatives of rapamycin, known as rapalogs, have the same molecular scaffold but different physiochemical properties. Clinical trials are ongoing with rapamycin, temsirolimus (Torisel, Wyeth), everolimus (Afinitor, Novartis) and deforolimus (ARIAD/Merck) in a wide range of human malignancies. Temsirolimus and everolimus have been approved by the FDA for the treatment of patients with advanced renal cell carcinoma ${ }^{[5,6]}$. Everolimus has also been approved as monotherapy or a component of combination therapy for pediatric and adult patients with subependymal giant cell astrocytoma (SEGA), progressive neuroendocrine tumors of pancreatic origin (PNET), and SEGA associated with tuberous sclerosis (TS) (http://www.cancer.gov/cancertopics/druginfo/fda-everolimus). The second generation of mTOR inhibitors are active-site inhibitors, which simultaneously inhibit mTORC1 and mTORC2 by interacting with the ATP pocket of the mTOR catalytic domain ${ }^{[7,8]}$. Numerous active-site inhibitors have been discovered and are currently in clinical trials, but few clinical results have been reported thus far.

Although rapalogs have validated the concept that the $\mathrm{PI} 3 \mathrm{~K} / \mathrm{Akt} / \mathrm{mTOR}$ pathway can be successfully targeted in clinical cancer treatment, objective responsive rates with rapa- 
logs have been modest and highly variable in most tumor types. One important strategy to improve the efficacy of rapa$\log$ is to identify predictive biomarkers that are capable of predicting the patients whom are most likely to benefit from treatment. Combinatorial use of rapalogs with other drugs is another promising strategy to improve treatment efficacy. This review will focus on results from recent clinical studies and strategies to improve the efficacy of rapalogs.

\section{Rapalogs in monotherapy}

To date, only temsirolimus and everolimus among the rapalogs have been approved for cancer therapy. Numerous clinical trials with rapamycin, temsirolimus, everolimus and deforolimus are ongoing in a wide range of human tumor types ${ }^{[9]}$. All of the rapalogs act similarly to inhibit mTORC1 and induce cell cycle arrest in $G_{1}$ phase.

\section{Sirolimus}

Sirolimus is the commercial name for rapamycin, a macrolide antibiotic discovered to have anticancer activity in 2002 . Numerous studies have been performed to demonstrate the anticancer activity of sirolimus in a wide range of malignancies. However, the results from clinical trials have been disappointing. Approximately 20 clinical trials of sirolimus in cancer therapy are currently active, and in most studies, sirolimus is being evaluated in combination with other drugs. Limitations in the desired pharmacological properties and lack of intellectual property protection have led pharmaceutical companies to develop sirolimus analogs, including temsirolimus and everolimus, with improved water solubility.

\section{Temsirolimus}

Temsirolimus is an ester analog of rapamycin with improved pharmaceutical properties, including stability and solubility. Temsirolimus is formulated for intravenous injection. Although temsirolimus itself possesses anticancer activity, it is converted into rapamycin in vivo. Temsirolimus was approved by the U. S. Food and Drug Administration (FDA) in May 2007 for the treatment of advanced renal cell carcinoma ${ }^{[1]}$ and was also approved by the European Medicines Agency (EMEA) in November 2007. Temsirolimus monotherapy also exerts significant efficacy in mantle cell lymphoma (MCL) and was approved for the treatment of relapsed and/or refractory MCL in the European Union in 2012. Currently, temsirolimus is undergoing evaluation as a monotherapy for the treatment of lymphoma and as a component of combination regimens in most clinical studies for the treatment of neuroblastoma, advanced hepatocellular carcinoma, etc. (http://www.cancer. gov/clinicaltrials).

\section{Everolimus}

Everolimus is the 40-O-(2-hydroxyethyl) derivative of rapamycin. Everolimus is orally available and is itself the main circulating component in human blood. Everolimus has been approved by FDA for the treatment of various malignancies, including advanced hormone receptor-positive and HER2negative breast cancer in combination with exemestane, pediatric and adult patients with SEGA, PNET, and SEGA associated with TS. Everolimus is being studied in phase I III clinical trials for the treatment of advanced hepatocellular carcinoma as a single agent or for the treatment of various cancers, including gastric carcinoma and pancreatic cancer, in combination with additional therapies. Available results have demonstrated a lack of efficacy as a single agent in hepatocellular carcinoma after sorafenib progression ${ }^{[10]}$, which might be due to failure to stratify the patients in the trials. Moreover, numerous combination therapies with additional drugs have failed to achieve desirable results if the patients were not preselected, indicating that a biomarker-guided clinical trial is warranted to improve the success rates.

\section{Biomarkers predicting the efficacy of rapalogs in monotherapy}

Although rapalogs have been approved for several types of cancer, the efficacy as monotherapy is not as significant as desired. Therefore, the identification of predictive biomarkers of treatment response is important to improve the efficacy of rapalogs. Currently, two strategies are primarily employed to discover rapalog biomarkers: an mTOR pathway-based strategy and a non-biased, high-throughput screen of tumor cells or patient tissues treated with rapalogs.

\section{Pathway-based identification of predictive biomarkers}

Aberrations in the MTORC1 signaling pathway drives tumor initiation, growth and development. Because rapalogs are specific inhibitors of mTORC1, tumors with hyper-activated mTORC1 signaling are predicted to be more sensitive to rapalogs. Therefore, signaling molecules upstream or downstream of mTORC1, which may indicate the activation status of mTORC1, have been investigated for their utility as predictive biomarkers for rapalog efficacy.

\section{PI3K-PTEN-AKT}

PI3Ks activate downstream targets, including mTOR via Akt. PIK3CA, which encodes $\mathrm{p} 110 \mathrm{a}$, is frequently mutated in a wide variety of common human tumors ${ }^{[11]}$. Weigelt et al have demonstrated that breast cancer cells harboring PIK3CA mutations are selectively sensitive to everolimus ${ }^{[12]}$. Using a panel of isogenic breast epithelial cell lines, Di Nicolantonio et al have identified oncogenic variants of PIK3CA and KRAS as determinants of response to everolimus ${ }^{[13]}$. The authors have further shown that human cancer cells that originated from different tissue types and harbor alterations in the PI3K pathway are responsive to everolimus. However, cells are resistant to everolimus when KRAS mutations occur concomitantly or are exogenously introduced. Importantly, the authors found that the presence of oncogenic KRAS mutations is associated with a lack of benefit after everolimus therapy in a cohort of metastatic cancer patients ${ }^{[13]}$. A more recent clinical study of temsirolimus and ridaforolimus in endometrial cancer has discovered that either PIK3CA mutation or KRAS mutation 
correlates with response $^{[14]}$. Thus, although PIK3CA and KRAS mutations may predict response to rapalogs, the response may also depend on the tissue origin of the cancer.

PTEN (Phosphatase and tensin homolog) is a negative regulator of PI3K, which dephosphorylates the 3-phosphate on the second-messenger molecule phosphatidylinositol-3,4,5triphosphate (PIP3) and downregulates PI3K/mTOR signaling. A deficiency in PTEN activity activates PI3K and downstream signaling. PTEN is one of the most frequently mutated, deleted or silenced tumor suppressors in human cancer. Temsirolimus has been found to preferentially block the growth of PTEN-null cells ${ }^{[6]}$. PTEN-deficient myeloma cell lines have been found to be remarkably sensitive to temsirolimus compared to those harboring wild-type PTEN, while introduction of wild-type PTEN in PTEN-deficient myeloma cells is insufficient to restore drug sensitivity ${ }^{[15]}$. These data suggest that factors other than PTEN also affect rapalog activity. Wendel et al have found that loss of even one PTEN allele renders lymphomas sensitive to rapamycin, while introducing eIF4E or disabling apoptosis in $\mathrm{PTEN}^{+/-}$lymphomas promotes resistance to rapamycin ${ }^{[16]}$. These data further support the notion that PTEN might not be the sensitivity-limiting factor for rapalogs. Similarly, in a study using a panel of 31 breast cancer cells lines, the cell lines that lack functional PTEN have been shown to be insensitive to everolimus ${ }^{[12]}$. Yang et al have reported that PTEN loss fails to predict response to everolimus in a glioblastoma orthotopic xenograft test panel ${ }^{[17]}$. Results from clinical trials with everolimus for the treatment bladder cancer have indicated that PTEN loss might be associated with everolimus resistance ${ }^{[18]}$, which has been further confirmed by another study using bladder cancer cells and xenografts ${ }^{[19]}$. Together, these data show that loss of PTEN function in many tumors is insufficient to predict response to rapalogs in human cancers, and whether PTEN status predicts the sensitivity/ resistance to rapalogs remains controversial.

The serine/threonine kinase Akt functions as a critical mediator of signaling downstream of PI3K. Full activation of Akt requires phosphorylation at T308 by PDK1 and at $\mathrm{S} 473$ by mTORC2. Akt is well established as the predominant PI3K effector in many cell types, and phosphorylation of Akt is often considered to be surrogate readout of PI3K activity. Thus, phosphorylated Akt has been hypothesized to be a predictive biomarker for sensitivity to rapalogs. Gera et al have found that Akt activity determines sensitivity to rapamycin, which is associated with downregulation of cyclin D1 and c-Myc ${ }^{[20]}$. Phosphorylated Akt and overexpression of S6K1 have also been reported to be associated with rapamycin sensitivity in a panel of breast cancer cell lines ${ }^{[21]}$. Heightened Akt activity has been shown to sensitize small lung cancer cells to everolimus and enhance temsirolimus activity in multiple myeloma cells $^{[22,23]}$. A trend toward a positive association between the level of phosphorylated Akt in tumor samples and response to temsirolimus has been observed in a randomized phase II trial in advanced renal cell carcinoma, whereas patients lacking high expression of either phospho-S6 or phospho-Akt experienced an objective tumor response ${ }^{[24]}$. However, Akt could be phosphorylated as a result of PI3K activation or by mTORC2 upon feedback loop upon inhibition of mTORC1 by rapalogs. The role of Akt in the activity of rapalogs requires further investigation in additional clinical settings.

\section{Immediate upstream components of mTORC1}

mTORC1 is also regulated independent of PI3K/PTEN-Akt, and the immediate upstream components of mTORC1 may affect rapalog activity. TSC1/2 (tuberous sclerosis complex $1 / 2$ ) negatively regulates mTOR activity and is often mutated in cancer. Whole genome sequencing of a patient with metastatic bladder cancer who demonstrated an extraordinary response to everolimus revealed a loss of function mutation in $\operatorname{TSC1}^{[25]}$. The genome sequencing of 13 additional bladder cancer patients in the same trial of everolimus found that patients with mutated TSC1 achieved better response those with wildtype $T S C 1^{[25]}$. In another clinical study, a patient with metastatic anaplastic thyroid carcinoma was found to develop resistance after an extraordinary 18-month response ${ }^{[26]}$. Whole-exome sequencing of pretreatment and drug-resistant tumors revealed a nonsense mutation in TSC2 (which suggests a mechanism for the exquisite sensitivity to everolimus) and a mutation in mTOR that confers resistance to everolimus. These results suggest that TSC1/2 mutations may serve as predictive biomarkers for the efficacy of rapalogs in at least some types of cancer.

A recent study by our group discovered that Rab1, a small GTPase, acts as an oncogene and mTORC1 activator independent of PI3K ${ }^{[27]}$. Rab1A overexpression promotes mTORC1 signaling and oncogenic growth in an amino acidand mTORC1-dependent manner. In colorectal cancer (CRC), overexpression of Rab1A correlates with elevated mTORC1 signaling, tumor invasion, progression, poor prognosis, and rapamycin sensitivity. More importantly, CRC cells with elevated Rab1A expression are especially sensitive to rapamycin. Amino acids also activate mTORC1 through the Rag GTPases, and GATOR1 has recently been identified as a key negative regulator of the Rag GTPases ${ }^{[28]}$. mTORC1 is hyperactive in cancer cells with inactivating mutations in GATOR1, and these cells are hypersensitive to rapamycin. These studies suggest that regulators of mTOR via amino acids are potential determinants of rapalog efficacy.

\section{MTOR}

TOR2 mutations in yeast were found to confer rapamycin resistance approximately 20 years ago ${ }^{[29]}$. However, rapamycin-resistant mutations in mTOR in humans were not described until 2000, when Sato et al identified two different point mutations, S2215Y and R2505P, that cause constitutive mTORC1 activation in a human cancer genome database ${ }^{[30]}$. The mTOR mutants retain the ability to phosphorylate 4E-BP1 upon starvation and render sensitivity to rapamycin ${ }^{[30]}$. Grabiner et al later generated a comprehensive catalog of mTOR cancer mutations and found that 33 mTOR mutations confer mTORC1 pathway hyperactivation ${ }^{[31]}$. The cancer mutations cluster in six distinct regions in the C-terminal half of mTOR 
and occur in multiple cancer types. Cancer cell lines with hyperactivating mTOR mutations display enhanced sensitivity to rapamycin in both cultured cells and in vivo xenografts ${ }^{[3]}$. An exceptional responder in a phase I study of pazopanib and everolimus in advanced solid tumors has recently been identified $^{[32]}$. Whole-exome sequencing of the patient with a 14-month complete response on this trial revealed two concurrent activating mutations in $\mathrm{mTOR}^{[32]}$.

\section{Downstream Components of mTORC1}

Several molecules downstream of mTOR have also been studied as determinant factors of rapalog sensitivity, including the mTOR substrates S6K1 and 4EBP1 and the mTORregulated proteins, such as cyclin D1 and HIF-1a.

Phosphorylated S6K1 has been reported to be indicative of rapamycin sensitivity in human colorectal cancer cells ${ }^{[33]}$. In a phase II trial of temsirolimus in recurrent glioblastoma multiforme, high levels of phosphorylated S6K1 in baseline tumor samples appear to predict a patient population more likely to derive benefit from treatment ${ }^{[34]}$. As the substrate of S6K1, phosphorylated $\mathrm{S} 6$ ribosomal protein can indicate the activity of S6K1. The level of phosphorylated S6 ribosomal protein has been found to be predictive of early response to deforolimus in the treatment of advanced sarcoma ${ }^{[35]}$ and response to temsirolimus in advanced renal cell carcinoma ${ }^{[24]}$. Unfortunately, clinical trials of deforolimus for the treatment of sarcoma have been discontinued after marketing rejection by the FDA in June 2012.

High levels of 4E-BP1 have been reported to be associated with high-grade tumors and poor prognosis in patients with ovarian carcinoma ${ }^{[36]}$. By evaluating the expression of activated mTOR pathway components in 57 endometrial cancer surgical specimens, Darb-Esfahani et al have discovered that high levels of phosphorylated 4E-BP1 characterize high-grade, high-stage endometrial adenocarcinomas ${ }^{[37]}$. The authors also found that Ishikawa endometrial cells possessing hyper-phosphorylated 4E-BP1 are more sensitive to rapamycin than HEC$1 \mathrm{~A}$ cells with low levels of phosphorylated 4E-BP1. Because the sample size was rather small, further study is required to validate the potential of phosphorylated $4 \mathrm{E}-\mathrm{BP} 1$ as a predictive biomarker of rapalog efficacy.

The translation of cyclin D1 is under the control of an mTOR-mediated pathway, and rapalogs can downregulate the expression of cyclin D1 in many tumor types. Mantle cell lymphoma is characterized by overexpression of cyclin D1 via a $\mathrm{t}(11 ; 14)(\mathrm{q} 13 ; \mathrm{q} 32)$ chromosomal translocation. Temsirolimus has been shown to significantly improve progression-free survival and objective response in mantle cell lymphoma ${ }^{[38,39]}$, suggesting that cyclin D1 may be useful as a biomarker to predict the efficacy of rapalogs. However, whether cyclin D1 can predict rapalog efficacy in other tumor types requires further investigation.

Von Hippel-Lindau (VHL) is a tumor suppressor gene encoding an E3 ligase that promotes the ubiquitylation of the hypoxia-inducible transcription factors HIF1, HIF2 and HIF3, leading to their degradation by the proteasome. Loss of $V H L$ is found in approximately $50 \%-70 \%$ of renal cell carcinomas, in which high steady-state levels of HIF are expressed. HIF protein translation is dependent on an mTOR-mediated signaling cascade, which suggests a rationale that mTOR inhibitors could be used for the treatment of renal cell carcinoma. In fact, temsirolimus and everolimus have been approved for advanced renal carcinoma. Thomas et al have found that loss of VHL sensitizes kidney cancer cells to temsirolimus in vitro and in mouse models ${ }^{[40]}$, suggesting that alterations in VHL or expression of HIF1acould be used as predictive biomarkers for rapalog-based therapy in renal cell carcinoma ${ }^{[40,41]}$. However, a global phase III study with temsirolimus and interferonalpha in advanced renal cell carcinoma has found that the baseline status of PTEN and HIF1a does not correlate with efficacy in renal cell carcinoma patients treated with temsirolimus or interferon ${ }^{[42]}$. The lack of correlation may be due to the variability in tumor specimens that occurred as a result of the global nature of the clinical trial. Similar results suggesting no correlation between PTEN expression or VHL status and response to temsirolimus in a randomized phase II trial have been reported ${ }^{[24]}$. Thus, other biomarkers are needed to predict response to rapalogs in renal carcinoma patients.

\section{Omics-based approach to identify predictive biomarkers of rapalog efficacy}

As discussed above, several predictive biomarkers based on the PI3K pathway have been identified. However, these biomarkers are limited by current knowledge and have remained controversial. Novel high-throughput technologies for the analysis of global gene/protein expression profiles and gene mutations may expand the scope of biomarker candidates and improve the reliability of prediction.

To search for potential biomarkers that predict rapalog sensitivities, the correlations between rapalog activities and genes expression patterns in the NCI-60 cell lines have been studied $^{[43]}$. An analysis of 9706 gene probes has discovered that $C D K N 1 B$, which encodes the p27 protein, is the most highly positively correlated gene. The correlation between p27 expression and rapalog activity is likely determined by the basal level of p27, which may provide a putative threshold to block the $\mathrm{G}_{1} / \mathrm{S}$ transition upon rapalog treatment; this correlation has subsequently been confirmed in human breast cancer cells and human cancer xenograft ${ }^{[33]}$. Because everolimus has been approved for the treatment of postmenopausal women with advanced hormone receptor-positive, HER2-negative breast cancer in combination with exemestane, employing p27 as a predictive marker to predefine response to rapalogs or develop combination regimens would improve response rates to rapalog-based therapy in breast cancer. p27 levels have also been shown to predict the responsiveness of pancreatic cancer cells to the dual PI3K/mTOR inhibitor BEZ235 ${ }^{[44]}$. Further validation of $\mathrm{p} 27$ as a candidate predictive biomarker of rapalog response in prospective randomized clinical trials is warranted.

The European Union multi-disciplinary Personalized RNA interference to Enhance the Delivery of Individualized Cyto- 
toxic and Targeted therapeutics (PREDICT) consortium has recently initiated a framework to accelerate the development of predictive biomarkers of individual patient response to anticancer agents ${ }^{[45]}$. The consortium focuses on the identification of reliable predictive biomarkers to everolimus and sunitinib, a multi-targeted tyrosine kinase inhibitor. The PREDICT consortium planned to use established and novel methods to integrate clinical trials with high-throughput genomic analyses, including personalized tumor-derived small hairpin RNA and high-throughput small interfering RNA screens, to identify and validate functionally important genomic or transcriptomic predictive biomarkers of individual drug response in patients. Though clinical trials (E-PREDICT) using everolimus in renal cell carcinoma are currently undergoing, it may provide a good example to integration of personalized functional genomics into clinical setting, which may allow an unprecedented opportunity to identify patient-specific drug sensitivity pathways rather than be restricted to the known pathway context. Another advantage of this approach is that the analyses are performed in the clinical setting, circumventing the challenge of translating the data obtained from in vitro or in vivo studies to clinical implications.

\section{Combination therapy based on rapalogs}

This limited clinical efficacy of rapalogs may be due to insufficient inhibition of the mTORC1 substrates ${ }^{[46]}$, the well-known feedback Akt activation triggered by mTORC1 inhibition ${ }^{[4]}$, or the emergence of novel drug resistance mechanisms ${ }^{[48]}$. The combination of rapalogs with other anticancer drugs is an important strategy to improve their efficacy and has been studied in multiple preclinical and clinical settings ${ }^{[49,50]}$. A wide spectrum of drugs have been studied in combination with rapalogs, including conventional cytotoxic drugs and agents targeting the PI3K/Akt pathway and related parallel pathways.

\section{Combination with conventional cytotoxic drugs}

Rapalogs induce cytostatic effects instead of death in tumor cells. Combining rapalogs with conventional cytotoxic drugs significantly enhances their anticancer activity in preclinical studies. These encouraging results have pushed the studies into clinical settings. Currently, rapalogs are being tested in combination with platinum, topotecan, capecitabine, 5-Fluorouracil and mitomycin $\mathrm{C}$, etc. in various cancer types. In a recent study, the combination of everolimus and carboplatin was efficacious in metastatic triple negative breast cancer ${ }^{[51]}$. Although dose-limiting hematological toxicity was observed when AUC 5/6 of carboplatin was combined with everolimus, carboplatin AUC 4 was well-tolerated in combination with everolimus with continuing responses ${ }^{[51]}$. Therefore, achieving the balance between efficacy and toxicity is an important issue in developing the appropriate combination of rapalogs with cytotoxic drugs.

\section{Targeting multiple components within the pathway}

Because rapalogs inhibit only the activity of mTORC1 and induce feed-back activation of Akt, combining rapalogs with inhibitors targeting the components within the pathway (such as upstream growth factor receptors and PI3K) seems to be a promising strategy. Rapalogs have been tested extensively in combination with monoclonal antibodies targeting growth factors or receptors, such as dalotuzumab (anti-IGF1R), cetuximab (anti-EGFR), bevacizumab (anti-VEGFa), and tyrosine kinase inhibitors, such as sunitinib, sorafenib and the PI3K inhibitor BYL-719. The combination of everolimus with trastuzumab plus vinorelbine significantly prolongs progression-free survival inpatients with trastuzumab-resistant and taxane-pretreated, HER2-positive, advanced breast cancer ${ }^{[52]}$. However, the clinical benefit should be considered in the context of the adverse event profile in this population. The combination of temsirolimus and bevacizumab has demonstrated substantial activity and reasonable tolerability in pancreatic neuroendocrine tumor in a multicenter phase II trial, with RR of $41 \%$, which is well in excess of single targeted agents ${ }^{[53]}$.

\section{Targeting parallel pathways}

Tumor cells have evolved complicated signaling networks to support their survival and proliferation. Inhibition of mTOR could be compensated by parallel pathways. Simultaneously targeting mTOR and its parallel pathways is an attractive strategy to improve the efficacy of rapalogs. Rapalogs have been tested in combination with drugs regulating MEK, AMPK and autophagy. Although the combination of AZD6244 with rapamycin has been reported to display antitumor and antiangiogenic effects in preclinical models of human $\mathrm{HCC}^{[54]}$, a phase IB trial of the oral MEK inhibitor trametinib (GSK1120212) in combination with everolimus in patients with advanced solid tumors has revealed frequent treatmentrelated adverse events in treated patients ${ }^{[55]}$. Metformin is an activator of AMPK and leads to inhibition of mTOR, and clinical trials with the combination of rapalogs and metformin are ongoing.

\section{Future perspectives}

mTORC1 plays pivotal roles in multiple cellular processes and is aberrantly activated in cancers. Rapalogs have been shown to be promising therapeutics for various types of human malignancies. Great progress has been made in recent years to better understand the role and regulation of mTOR and its signaling network context. This progress has facilitated the identification of biomarker candidates to predict efficacy and combinatorial approaches for rapalog-based cancer therapy and ultimately to improve efficacy and expand the clinical use of rapalogs. However, rapalog-based cancer therapy is far from desirable and requires further optimization. To achieve this goal, the following issues should be addressed: 1) develop a more profound understanding of the regulation of mTORC1 in physiological and pathological conditions and the mechanism action of rapalogs; 2) identify biomarkers to stratify patients who are likely to benefit from rapalogs, which could be realized by large scale and high-throughput analyses of the correlations between gene background and rapalog efficacy in 
certain types of cancer in clinical settings; and 3) elucidate the mechanism of sensitivity/resistance to rapalogs in patients, which will help to constitute drug combinations; 4) because of the critical role of mTOR in immune modulation, combination of rapalogs with cancer immunotherapy is warranted to be studied in both preclinical and clinical settings. With the promise and implementation of precision medicine, further efforts are warranted to expand the therapeutic benefit of rapalogs in additional cancer patients.

\section{Acknowledgements}

This work was supported by NIH R01 grants CA123391, CA173519 and CA166575 to X.F. Steven ZHENG and by National Natural Science Foundation of China (81321092, 81173079, 81373445) to Ling-hua MENG.

\section{References}

1 Ma XM, Blenis J. Molecular mechanisms of mTOR-mediated translational control. Nat Rev Mol Cell Biol 2009; 10: 30-18.

2 Peterson TR, Laplante M, Thoreen CC, Sancak Y, Kang SA, Kuehl WM, et al. DEPTOR is an mTOR inhibitor frequently overexpressed in multiple myeloma cells and required for their survival. Cell 2009; 137: 873-86.

3 Hara K, Maruki Y, Long XM, Yoshino K, Oshiro N, Hidayat S, et al. Raptor, a binding partner of target of rapamycin (TOR), mediates TOR action. Cell 2002; 110: 177-89.

4 Sarbassov DD, Ali SM, Kim DH, Guertin DA, Latek RR, ErdjumentBromage $\mathrm{H}$, et al. Rictor, a novel binding partner of mTOR, defines a rapamycin-insensitive and raptor-independent pathway that regulates the cytoskeleton. Curr Biol 2004; 14: 1296-302.

5 Hudes G, Carducci M, Tomczak P, Dutcher J, Figlin R, Kapoor A, et al. Temsirolimus, interferon alfa, or both for advanced renal-cell carcinoma. N Engl J Med 2007; 356: 2271-81.

6 Motzer RJ, Escudier B, Oudard S, Hutson TE, Porta C, Bracarda S, et al. Efficacy of everolimus in advanced renal cell carcinoma: a doubleblind, randomised, placebo-controlled phase III trial. Lancet 2008; 372: 449-56.

7 Garcia-Echeverria C. Allosteric and ATP-competitive kinase inhibitors of mTOR for cancer treatment. Bioorg Med Chem Lett 2010; 20 : 4308-12.

8 Zhang Y, Zheng XF. mTOR-independent 4E-BP1 phosphorylation is associated with cancer resistance to mTOR kinase inhibitors. Cell cycle 2012; 11: 594-603.

9 Don AS, Zheng XF. Recent clinical trials of mTOR-targeted cancer therapies. Rev Recent Clin Trials 2011; 6: 24-35.

10 Llovet JM, Hernandez-Gea V. Hepatocellular carcinoma: reasons for phase III failure and novel perspectives on trial design. Clin Cancer Res 2014; 20: 2072-9.

11 Samuels Y, Waldman T. Oncogenic mutations of PIK3CA in human cancers. Curr Top Microbiol Immunol 2010; 347: 21-41.

12 Weigelt B, Warne PH, Downward J. PIK3CA mutation, but not PTEN loss of function, determines the sensitivity of breast cancer cells to mTOR inhibitory drugs. Oncogene 2011; 30: 3222-33.

13 Di Nicolantonio F, Arena S, Tabernero J, Grosso S, Molinari F, Macarulla T, et al. Deregulation of the PI3K and KRAS signaling pathways in human cancer cells determines their response to everolimus. J Clin Invest 2010; 120: 2858-66.

14 Mackay HJ, Eisenhauer EA, Kamel-Reid S, Tsao M, Clarke B, Karakasis $\mathrm{K}$, et al. Molecular determinants of outcome with mammalian target of rapamycin inhibition in endometrial cancer. Cancer 2014; 120 : 603-10.

15 Shi Y, Gera J, Hu L, Hsu JH, Bookstein R, Li W, et al. Enhanced sensitivity of multiple myeloma cells containing PTEN mutations to CCl-779. Cancer Res 2002; 62: 5027-34.

16 Wendel HG, Malina A, Zhao Z, Zender L, Kogan SC, Cordon-Cardo $\mathrm{C}$, et al. Determinants of sensitivity and resistance to rapamycinchemotherapy drug combinations in vivo. Cancer Res 2006; 66: 7639-46.

17 Yang L, Clarke MJ, Carlson BL, Mladek AC, Schroeder MA, Decker P, et al. PTEN loss does not predict for response to RAD001 (Everolimus) in a glioblastoma orthotopic xenograft test panel. Clin Cancer Res 2008; 14: 3993-4001.

18 Seront E, Rottey S, Sautois B, Kerger J, D'Hondt LA, Verschaeve V, et al. Phase II study of everolimus in patients with locally advanced or metastatic transitional cell carcinoma of the urothelial tract: clinical activity, molecular response, and biomarkers. Ann Oncol 2012; 23: 2663-70.

19 Seront E, Pinto A, Bouzin C, Bertrand L, Machiels JP, Feron O. PTEN deficiency is associated with reduced sensitivity to mTOR inhibitor in human bladder cancer through the unhampered feedback loop driving PI3K/Akt activation. Br J Cancer 2013; 109: 1586-92.

20 Gera JF, Mellinghoff IK, Shi Y, Rettig MB, Tran C, Hsu JH, et al. AKT activity determines sensitivity to mammalian target of rapamycin (mTOR) inhibitors by regulating cyclin D1 and c-myc expression. J Biol Chem 2004; 279: 2737-46.

21 Noh WC, Mondesire WH, Peng J, Jian W, Zhang H, Dong J, et al. Determinants of rapamycin sensitivity in breast cancer cells. Clin Cancer Res 2004; 10 (3): 1013-23.

22 Marinov M, Ziogas A, Pardo OE, Tan LT, Dhillon T, Mauri FA, et al. $\mathrm{AKT} / \mathrm{mTOR}$ pathway activation and $\mathrm{BCL}-2$ family proteins modulate the sensitivity of human small cell lung cancer cells to RAD001. Clin Cancer Res 2009; 15: 1277-87.

23 Frost $\mathrm{P}$, Shi $\mathrm{Y}$, Hoang $\mathrm{B}$, Lichtenstein A. AKT activity regulates the ability of mTOR inhibitors to prevent angiogenesis and VEGF expression in multiple myeloma cells. Oncogene 2007; 26: 2255-62.

24 Cho D, Signoretti S, Dabora S, Regan M, Seeley A, Mariotti M, et al. Potential histologic and molecular predictors of response to temsirolimus in patients with advanced renal cell carcinoma. Clin Genitourin Cancer 2007; 5: 379-85.

25 Iyer G, Hanrahan AJ, Milowsky MI, Al-Ahmadie H, Scott SN, Janakiraman $\mathrm{M}$, et al. Genome sequencing identifies a basis for everolimus sensitivity. Science 2012; 338: 221.

26 Wagle N, Grabiner BC, Van Allen EM, Amin-Mansour A, Taylor-Weiner A, Rosenberg $\mathrm{M}$, et al. Response and acquired resistance to everolimus in anaplastic thyroid cancer. N Engl J Med 2014; 371: 1426-33.

27 Thomas JD, Zhang YJ, Wei YH, Cho JH, Morris LE, Wang HY, et al. Rab1A is an mTORC1 activator and a colorectal oncogene. Cancer cell 2014; 26: 754-69.

28 Bar-Peled L, Chantranupong L, Cherniack AD, Chen WW, Ottina KA, Grabiner BC, et al. A Tumor suppressor complex with GAP activity for the Rag GTPases that signal amino acid sufficiency to mTORC1. Science 2013; 340: 1100-6.

29 Lorenz MC, Heitman J. TOR mutations confer rapamycin resistance by preventing interaction with FKBP12-rapamycin. J Biol Chem 1995; 270: $27531-7$.

30 Sato T, Nakashima A, Guo L, Coffman K, Tamanoi F. Single amino-acid changes that confer constitutive activation of mTOR are discovered in human cancer. Oncogene 2010; 29: 2746-52.

31 Grabiner BC, Nardi V, Birsoy K, Possemato R, Shen K, Sinha S, et al. A diverse array of cancer-associated MTOR mutations are 
hyperactivating and can predict rapamycin sensitivity. Cancer Discov 2014; 4: 554-63.

32 Wagle N, Grabiner BC, Van Allen EM, Hodis E, Jacobus S, Supko JG, et al. Activating mTOR mutations in a patient with an extraordinary response on a phase I trial of everolimus and pazopanib. Cancer Discov 2014; 4: 546-53.

33 Nozawa H, Watanabe T, Nagawa H. Phosphorylation of ribosomal p70 S6 kinase and rapamycin sensitivity in human colorectal cancer. Cancer Lett 2007; 251: 105-13.

34 Galanis E, Buckner JC, Maurer MJ, Kreisberg J, Ballman K, Boni J, et al. Phase II trial of temsirolimus (CCl-779) in recurrent glioblastoma multiforme: a North Central Cancer Treatment Group Study. J Clin Oncol 2005; 23: 5294-304.

35 Iwenofu OH, Lackman RD, Staddon AP, Goodwin DG, Haupt HM, Brooks JS. Phospho-S6 ribosomal protein: a potential new predictive sarcoma marker for targeted mTOR therapy. Mod Pathol 2008; 21: 231-7.

36 Castellvi J, Garcia A, Rojo F, Ruiz-Marcellan C, Gil A, Baselga J, et al. Phosphorylated $4 \mathrm{E}$ binding protein 1: a hallmark of cell signaling that correlates with survival in ovarian cancer. Cancer 2006; 107: 180111.

37 Darb-Esfahani S, Faggad A, Noske A, Weichert W, Buckendahl AC, Muller B, et al. Phospho-mTOR and phospho-4EBP1 in endometrial adenocarcinoma: association with stage and grade in vivo and link with response to rapamycin treatment in vitro. J Cancer Res Clin Oncol 2009; 135: 933-41.

38 Hess G, Herbrecht R, Romaguera J, Verhoef G, Crump M, Gisselbrecht $\mathrm{C}$, et al. Phase III study to evaluate temsirolimus compared with investigator's choice therapy for the treatment of relapsed or refractory mantle cell lymphoma. J Clin Oncol 2009; 27: 3822-9.

39 Witzig TE, Geyer SM, Ghobrial I, Inwards DJ, Fonseca R, Kurtin P, et al. Phase II trial of single-agent temsirolimus (CCl-779) for relapsed mantle cell lymphoma. J Clin Oncol 2005; 23: 5347-56.

40 Thomas GV, Tran C, Mellinghoff IK, Welsbie DS, Chan E, Fueger B, et al. Hypoxia-inducible factor determines sensitivity to inhibitors of mTOR in kidney cancer. Nat Med 2006; 12: 122-7.

41 Gossage L, Eisen T. Alterations in VHL as potential biomarkers in renal-cell carcinoma. Nat Rev Clin Oncol 2010; 7: 277-88.

42 Figlin RA, de Souza P, McDermott D, Dutcher JP, Berkenblit A, Thiele A, et al. Analysis of PTEN and HIF-1alpha and correlation with efficacy in patients with advanced renal cell carcinoma treated with temsirolimus versus interferon-alpha. Cancer 2009; 115: 3651-60.

43 Chen G, Yang N, Wang X, Zheng SY, Chen Y, Tong LJ, et al. Identification of p27/KIP1 expression level as a candidate biomarker of response to rapalogs therapy in human cancer. J Mol Med (Berl) 2010; 88: 941-52.

44 Diersch S, Wenzel P, Szameitat M, Eser P, Paul MC, Seidler B, et al. Efemp1 and p27(Kip1) modulate responsiveness of pancreatic cancer cells towards a dual PI3K/mTOR inhibitor in preclinical models. Oncotarget 2013; 4: 277-88.

45 Swanton C, Larkin JM, Gerlinger M, Eklund AC, Howell M, Stamp G, et al. Predictive biomarker discovery through the parallel integration of clinical trial and functional genomics datasets. Genome Med 2010; 2: 53.

46 Choo AY, Yoon SO, Kim SG, Roux PP, Blenis J. Rapamycin differentially inhibits S6Ks and 4E-BP1 to mediate cell-type-specific repression of mRNA translation. Proc Natl Acad Sci U S A 2008; 105: 17414-9.

47 O'Reilly KE, Rojo F, She Q-B, Solit D, Mills GB, Smith D, et al. mTOR inhibition induces upstream receptor tyrosine kinase signaling and activates Akt. Cancer Res 2006; 66: 1500-8.

48 Huang J, Manning BD. A complex interplay between Akt, TSC2, and the two mTOR complexes. Biochem Soc Trans 2009; 37: 217.

49 Ghayad SE, Cohen PA. Inhibitors of the PI3K/Akt/mTOR pathway: new hope for breast cancer patients. Recent Pat Anticancer Drug Discov 2010; 5: 29-57.

50 Buck E, Eyzaguirre A, Brown E, Petti F, McCormack S, Haley JD, et al. Rapamycin synergizes with the epidermal growth factor receptor inhibitor erlotinib in non-small-cell lung, pancreatic, colon, and breast tumors. Mol Cancer Ther 2006; 5: 2676-84.

51 Singh J, Novik Y, Stein S, Volm M, Meyers M, Smith J, et al. Phase 2 trial of everolimus and carboplatin combination in patients with triple negative metastatic breast cancer. Breast Cancer Res 2014; 16: R32.

52 Andre F, O'Regan R, Ozguroglu M, Toi M, Xu B, Jerusalem G, et al. Everolimus for women with trastuzumab-resistant, HER2-positive, advanced breast cancer (BOLERO-3): a randomised, double-blind, placebo-controlled phase 3 trial. Lancet Oncol 2014; 15: 580-91.

53 Hobday TJ, Qin R, Reidy-Lagunes D, Moore MJ, Strosberg J, Kaubisch A, et al. Multicenter Phase II Trial of Temsirolimus and Bevacizumab in Pancreatic Neuroendocrine Tumors. J Clin Oncol 2015; 33:1551-6.

54 Huynh H. AZD6244 (ARRY-142886) enhances the antitumor activity of rapamycin in mouse models of human hepatocellular carcinoma. Cancer 2010; 116: 1315-25.

55 Tolcher AW, Bendell JC, Papadopoulos KP, Burris HA, 3rd, Patnaik A, Jones SF, et al. A phase IB trial of the oral MEK inhibitor trametinib (GSK1120212) in combination with everolimus in patients with advanced solid tumors. Ann Oncol 2015; 26: 58-64. 